\title{
The impact of nutritional risk factors and sarcopenia on survival in patients treated with pelvic exenteration for recurrent gynaecological malignancy: a retrospective cohort study
}

\author{
Veronika Seebacher ${ }^{1,2}$ (1) $\cdot$ Andrea Rockall ${ }^{3} \cdot$ Marielle Nobbenhuis $^{1} \cdot$ S. Aslam Sohaib ${ }^{3} \cdot$ Thomas Knogler $^{4}$. \\ Rosa M. Alvarez ${ }^{1}$. Desiree Kolomainen ${ }^{5} \cdot$ John H. Shepherd ${ }^{1} \cdot$ Clare Shaw $^{6}$. Desmond P. Barton ${ }^{1}$
}

Received: 5 October 2019 / Accepted: 24 September 2021 / Published online: 3 November 2021

(c) The Author(s) 2021

\begin{abstract}
Purpose The aim of the present study is to investigate the prognostic significance of nutritional risk factors and sarcopenia on the outcome of patients with recurrent gynaecological malignancies treated by pelvic exenteration.

Methods We retrospectively evaluated muscle body composite measurements based on pre-operative CT scans, nutritional risk factors as assessed by a validated pre-operative questionnaire, and clinical-pathological parameters in 65 consecutive patients with recurrent gynaecological malignancies, excluding ovarian cancer, treated by pelvic exenteration at the Royal Marsden Hospital London. Predictive value for postoperative morbidity was investigated by logistic regression analyses. Relevant parameters were included in uni- and multivariate survival analyses.

Results We found only (1) low muscle attenuation (MA) — an established factor for muscle depletion —and (2) moderate risk for malnutrition to be independently associated with shorter overall survival ( $p=0.006$ and $p=0.008$, respectively). MA was significantly lower in overweight and obese patients $(p=0.04)$. Muscle body composite measurements were not predictive for post-operative morbidity.

Conclusion The study suggests that pre-operative low MA and moderate risk for malnutrition are associated with shorter survival in patients with recurrent gynaecological malignancies treated with pelvic exenteration. Further studies are needed to validate these findings in larger cohorts.
\end{abstract}

Keywords Cachexia $\cdot$ Malnutrition $\cdot$ Muscle attenuation $\cdot$ Cervical cancer $\cdot$ Endometrial cancer $\cdot$ Vulvar cancer

\section{Introduction}

Pelvic exenteration is the last therapeutic option in selected, heavily pre-treated patients with pelvic recurrent or persistent gynaecological malignancies. Due to improvements in

Preliminary results of this study have been presented as posters at the following scientific meetings and have been published as abstracts in the respective meeting journal [36-38]. XXVI. Wissenschaftliche Tagung der Arbeitsgemeinschaft für Gynäkologische Onkologie (AGO) der OEGGG 2017, 27-29 April 2017 Salzburg, Austria. Proceedings of the International Cancer Imaging Society (ICIS) 17th Annual Teaching Course, 02-04 October 2017, Berlin, Germany. International meeting of the European Society of Gynaecological Oncology (ESGO), 04-07 November 2017; Vienna, Austria.

Veronika Seebacher

veronika.seebacher@meduniwien.ac.at

Extended author information available on the last page of the article surgical techniques, perioperative management and patient selection, postoperative 5 year overall survival (OS) rates ranging from 20 to $73 \%$ can be achieved while postoperative mortality rates have decreased from $20 \%$ to less than 5\% [1-8]. However, pelvic exenteration remains a morbid intervention with approximately $50 \%$ of patients suffering a major perioperative complication $[3-6,9,10]$. Therefore, patients are selected who are most likely to benefit and who are physically and psychologically fit enough to tolerate pelvic exenteration.

Over the past decade, cachexia, a multifactorial syndrome characterized by systemic inflammation and hypermetabolism, involuntary weight loss, and loss of skeletal muscle mass, and its impact on adverse clinical outcome in cancer patients has been extensively investigated [11-18]. Cachexia has been estimated to affect approximately $50 \%$ of all cancer patients and to account for up to $20 \%$ of cancer deaths [19]. 
One of its key components is sarcopenia, a syndrome characterised by progressive loss of skeletal muscle mass and strength. While sarcopenia is most commonly observed in older people, it can also develop secondary to prolonged immobility, inadequate dietary intake, malabsorption, inflammatory disease, or malignancy [20]. Both the presence of cancer and its treatment tend to disrupt homeostasis and lead to important metabolic changes to maintain homeostasis [19]. Computed tomography (CT) imaging does not directly measure cachexia. However, it can be used to estimate both the muscle mass and the muscle density. This is done by assessing the lumbar skeletal muscle area, by calculating the muscle attenuation (MA), a measure for muscle density, with lower values reflecting increased muscular lipid content $[11,18]$, and subsequently by calculating the skeletal muscle index (SMI). Both reduction of muscle mass and loss of muscle density have been associated with shorter survival in various malignancies [12-16], decreased tolerance to anti-cancer treatment [17, 21], and increased postoperative morbidity [22, 23].

The aim of the present study is to investigate the effect of nutritional risk factors, as assessed in a questionnaire, and body composition, i.e. skeletal muscle mass and MA assessed by $\mathrm{CT}$ imaging, on postoperative morbidity and survival in patients undergoing pelvic exenteration for recurrent or persistent gynaecological malignancies.

\section{Materials and methods}

\section{Patients' cohort and data acquisition}

We included patients who underwent pelvic exenteration for recurrent or persistent gynaecological malignancy at the Gynaecological Oncology Unit of the Royal Marsden Hospital (RMH), London, between 2000 and 2015. Clinical, pathological and radiological data were extracted retrospectively from electronic and paper-based medical records. Patients were excluded from analyses if the primary tumour was of ovarian origin, and/or if surgical data were missing or incomplete (see supplementary Fig. 1).

\section{Clinical management}

Prior to pelvic exenteration, clinical and radiological assessment was performed including an examination under anaesthesia, including diagnostic laparoscopy to evaluate operability [24]. Patients were only eligible for surgery if extrapelvic disease was excluded and if there was the expectation of achieving an $\mathrm{R} 0$ resection. We used the American Society of Anesthesiologists (ASA) physical status classification system and the age adjusted Charlson Comorbidity Index (AACCI) to assess performance status and perioperative risks, respectively [25, 26]. Postoperative morbidity and mortality were assessed within 60 days after pelvic exenteration and was classified according to the Clavien-Dindo classification of surgical complications [27]. We defined progression-free survival (PFS) as the date of first clinical or radiological evidence of progressive disease.

\section{Anthropometric measurements}

Height $(\mathrm{cm})$ and weight $(\mathrm{kg})$ prior to pelvic exenteration were annotated in the majority of patients during pre-assessment. Since 2008, risk factors for malnutrition and weight history were assessed routinely using the Royal Marsden Nutrition Screening Tool (RMNST) [28]. The RMNST is a nutritional screening tool developed by the Department of Nutrition and Dietetics of the RMH for inpatient use. It has been designed and validated to assess the risk of malnutrition at any time point in an oncology inpatient setting. In addition to the assessment of weight loss and food intake habits, it incorporates parameters characteristically affecting cancer patients such as mucositis, nausea, vomiting and dysphagia. The questionnaire allows stratification of patients into three risk groups for malnutrition: "low risk" (score of $0-4$ ), "medium risk" (score of 5-9), and "high risk" (score of $10+)$ [27].

\section{CT image analysis}

Only CT scans performed within 2 months prior to pelvic exenteration were used for analyses. CT scans were analysed by two independent researchers (VS and AR) trained in radiologic anatomy and body composition analysis, blinded to the outcome of the surgery. Two adjacent axial images within the same series, at the level of the third lumbar vertebra, were identified. Using predefined Hounsfield Unit (HU) ranges, the total cross-sectional areas $\left(\mathrm{cm}^{2}\right)$ of skeletal muscle tissue ( -29 to $150 \mathrm{HU})$, including the rectus abdominis, transversus abdominis, internal and external obliques, psoas, quadratus lumborum, and erector spinae muscles, the visceral adipose tissue (VAT; -150 to -50 $\mathrm{HU}$ ), and the subcutaneous adipose tissue (SAT; -190 to $-30 \mathrm{HU}$ ) were determined and analysed using Slice-OMatic software as previously described (v5.0, Tomovision, Montreal, Quebec, Canada) [11, 29]. In addition, the mean MA, was assessed by calculating the average $\mathrm{HU}$ value of the total muscle cross-sectional area. Values for cross-sectional surface of muscle (MS), visceral fat (VAT), subcutaneous fat (SAT), and muscle attenuation (MA) were averaged for each patient using two adjacent axial images within the same series at the level of the third lumbar vertebra. Subsequently, they were normalized for height in meters squared $\left(\mathrm{m}^{2}\right)$ and reported as lumbar skeletal muscle index (SMI; $\mathrm{cm}^{2} / \mathrm{m}^{2}$ ), lumbar visceral adipose tissue index (VATI; 
$\mathrm{cm}^{2} / \mathrm{m}^{2}$ ) and subcutaneous adipose tissue index (SATI; $\mathrm{cm}^{2} /$ $\mathrm{m}^{2}$ ), respectively.

\section{Statistical analysis}

Values are given as medians (interquartile range [IQR]). Differences in patients' characteristics were analysed using the Pearson Chi-Square Test. The Mann-Whitney $U$ test was used to compare medians of MA and MS between risk groups of clinico-pathological parameters, i.e. age $(<$ vs. $\geq$ the median age of 56 years), BMI ( $<$ vs. $\geq 25 \mathrm{~kg} /$ $\mathrm{m}^{2}$ ), and nutritional assessment according to the RMNST (low vs. moderate risk for malnutrition). We used $41.0 \mathrm{~cm}^{2} /$ $\mathrm{m}^{2}$ as a cut off for SMI as previously described [11]. As the number of patients in the present study was too small to generate cut-off values by optimum stratification for MA, VATI and SATI, we used the quartiles of these values to generate risk groups. Cut-off values were set at the lowest quartile for MA, and at the highest quartile for VATI and SATI. Survival probabilities were calculated by the product limit method of Kaplan and Meier. Differences between groups were tested using the log-rank test. The results were analysed for the endpoints of progression-free survival and overall survival (i.e. time between pelvic exenteration and date of disease recurrence/progression or date of death due to all causes, respectively). Patients alive with no or stable disease were censored with the date of last follow-up. Uniand multivariate Cox regression models were performed, comprising all variables that generated a $p$ value of $<0.05$ in univariate analysis.

Results of uni- and multivariate survival analyses are given as $p$ value [hazard-ratio (HR)])and 95\% confidence interval $(95 \% \mathrm{CI})$. Logistic regression analyses were performed to assess the predictive value of MA, SMI, VATI, and SATI risk groups and other clinical-pathological parameters for severe 60 days postoperative morbidity (i.e. Clavien-Dindo grade $\geq 3$ ). $p$ values $<0.05$ were considered statistically significant and all tests are two-sided. We used the statistical software SPSS 22.0 for Mac (SPSS 22.0.0, SPSS Inc., Chicago, IL) for statistical analysis.

\section{Results}

\section{Patients' characteristics}

A total of 76 patients underwent pelvic exenteration for recurrent or persistent gynaecological malignancy at the RMH from 2000 to 2015. After exclusion of patients with ovarian cancer and those in whom essential data elements were missing, 65 patients could be included into the present study. A flowchart depicting the patient selection process is shown in supplementary Fig. 1 (S1). Indications for pelvic exenteration were recurrent/persistent cancer of the vulva/vagina in $25(38.5 \%)$ patients, of the cervix uteri in $31(47.7 \%)$, and of the uterus in $9(13.8 \%)$. Histological subtypes of the primary cancer were adenocarcinoma in 17 patients (26.2\%), squamous cell carcinoma in 40 (61.5\%), clear cell carcinoma in $3(4.6 \%)$, mixed mullerian tumour in $1(1.5 \%)$, and sarcoma in $4(6.2 \%)$. Fifty-six patients $(86.2 \%)$ had radiotherapy in the past, $30(46.2 \%)$ had 2 or more previous lines of treatment. No patient received parenteral nutrition (PN) pre-operatively. Patients' characteristics at the time of pelvic exenteration are listed in Table 1.

Table 1 Characteristics and body composition measurements in 65 patients treated with pelvic exenteration for gynaecological malignancy

\begin{tabular}{|c|c|c|}
\hline & $n$ or median & $\%$ or IQR \\
\hline Median age (IQR) in years & 55 & $44-64$ \\
\hline \multicolumn{3}{|l|}{ ECOG } \\
\hline $0-1$ & 54 & 83.1 \\
\hline $2-3$ & 4 & 6.2 \\
\hline NA & 7 & 10.7 \\
\hline \multicolumn{3}{|l|}{ ASA } \\
\hline $0-1$ & 14 & 21.5 \\
\hline $2-3$ & 35 & 55.4 \\
\hline NA & 15 & 23.1 \\
\hline \multicolumn{3}{|l|}{ AACCI } \\
\hline 2 & 19 & 29.2 \\
\hline 3 & 13 & 20 \\
\hline 4 & 10 & 15.4 \\
\hline 5 & 13 & 20 \\
\hline 6 & 4 & 6.2 \\
\hline 7 & 1 & 1.5 \\
\hline NA & 5 & 7.7 \\
\hline \multicolumn{3}{|l|}{ Sarcopenia measurements $(n=32)$} \\
\hline $\begin{array}{l}\text { Median lumbar total muscle cross- } \\
\text { sectional area }\left(\mathrm{cm}^{2}\right)\end{array}$ & 113.8 & $104.9-129.7$ \\
\hline Median SMI $\left(\mathrm{cm}^{2} / \mathrm{m}^{2}\right)$ & 43.6 & $40.1-50.9$ \\
\hline $\mathrm{SMI}<41 \mathrm{~cm}^{2} / \mathrm{m}^{2}$ & 8 & 25.0 \\
\hline Median MA (HU) & 40.5 & $31.1-44.3$ \\
\hline $\mathrm{MA}<31 \mathrm{HU}$ & 7 & 20.6 \\
\hline Median VATI $\left(\mathrm{cm}^{2} / \mathrm{m}^{2}\right)$ & 87.4 & $67.5-120.9$ \\
\hline VATI $>120 \mathrm{~cm}^{2} / \mathrm{m}^{2}$ & 8 & 23.5 \\
\hline Median SATI $\left(\mathrm{cm}^{2} / \mathrm{m}^{2}\right)$ & 33.3 & $14.6-57.1$ \\
\hline SATI $>57 \mathrm{~cm}^{2} / \mathrm{m}^{2}$ & 8 & 23.5 \\
\hline
\end{tabular}

$I Q R$ Interquartile Range, ECOG Eastern Co-operative Oncology Group Performance Status, ASA American Society of Anesthesiologists risk score, AACCI Age Adjusted Charlson Comorbidity Index, SMI Skeletal Muscle Index, MA Muscle Attenuation, $H U$ Hounsfield Unit, VATI Visceral Adipose Tissue Index, SATI Subcutaneous Adipose Tissue Index 


\section{Surgical details and postoperative morbidity}

Thirty-one patients (47.7\%) underwent total exenteration, 20 (30.8\%) anterior, and $14(21.5 \%)$ posterior. Translevator and supralevator exenterations were performed in $41(63.1 \%)$ and in $24(36.9 \%)$ patients, respectively. In 7 patients $(10.8 \%)$, pelvic side wall resection was performed en-bloc with the pelvic exenteration. Plastic reconstruction of the pelvic floor with or without reconstruction of the vulva, vagina, and/or perineum was performed in 32 patients $(49.2 \%)$ and omental transposition in $42(64.6 \%)$. Forty patients $(61.5 \%)$ underwent 1 bowel anastomosis, and 4 (6.2\%). Surgical resection with margins free of tumour (R0 resection) was achieved in 37 (56.9\%) patients. In seven patients, detailed information on histological margins was missing. A median (IQR) duration of $610 \mathrm{~min}(475-740)$ and a median (IQR) number of four blood units (2-8) were required. Median (IQR) postoperative stay was 3 days (2-5) in the intensive care unit and 21 days (16-33) in the hospital. In 32 patients (49.2\%), PN was given for a median (IQR) of 8 (6-12) days postoperatively. A total of 16 patients (24.6\%) experienced Clavien-Dindo grade $\geq 3$ postoperative morbidity within 60 days after pelvic exenteration. No 60 day post-operative mortality occurred.

\section{Body composition measurements}

The median (IQR) BMI in our study population was $26.1(23.6-32.9) \mathrm{kg} / \mathrm{m}^{2}$. Only two patients $(3.1 \%)$ had a $\mathrm{BMI}<18.5$ and were therefore classified as underweight. Yet, data on BMI were missing in 19 patients. Seventeen patients $(26.2 \%)$ had mild hypoalbuminemia with serum albumin levels between 2.5 and $3.49 \mathrm{~g} / \mathrm{dl}$. No patient had severe hypoalbuminemia.

The RMNST was completed prior to pelvic exenteration in 34 patients which revealed that $26(76.5 \%)$ had low risk and $8(23.5 \%)$ moderate risk for malnutrition. No patient had high risk for malnutrition prior to pelvic exenteration.

CT scans were performed in 54 patients prior to pelvic exenteration, and in 32 images cases were digitally archived and these were then subjected to sarcopenia analyses. We compared the group of patients used for sarcopenia analyses with the rest of patients and did not find any differences between the two groups with regard to patients' age at time of surgery $(p=0.6)$, ECOG performance status $(p=0.1)$, type of pelvic exenteration $(p=0.2)$, postoperative need for PN $(p=0.5)$, severe 60 days postoperative morbidity $(p=0.5)$, the occurrence of disease recurrence $(p=0.1)$, or the patients' status at time point of last follow-up $(p=0.4)$. There were two patients who received chemotherapy within 60 days prior to the CT scan, one 10 days and the other one 33 days prior to CT scan. Both patients had high muscle attenuation. No other patients received chemotherapy or radiotherapy within 60 days prior to CT scan. Detailed clinical-pathological information in this group of patients is listed in supplementary Table 1.

Results of body composite measurements are shown in Table 1 . Overweight and obese patients had a significantly lower MA compared to under- and normal-weight patients. The medians (IQR) of SMI, MA, VATI, and SATI for clinical risk groups are shown in Table 2. Examples for sarcopenia measurements in two patients with high MA, and in two patients with low MA are depicted in Fig. 1.

We compared the medians of serum albumin between patients with SMI and MA below and above $41 \mathrm{~cm}^{2} / \mathrm{m}^{2}$ and $31 \mathrm{HU}$ (i.e., 25th percentile), respectively. We found no difference of median serum albumin levels between the respective groups of SMI and MA ( $p=0.5$ and $p=0.4$, respectively). In logistic regression analyses, none of the body composition measurements were predictive of severe 60-day postoperative morbidity (i.e., Clavien-Dindo grade $\geq 3$; see Table 3).

Table 2 Comparison of radiological body composition measurements between clinical risk groups $(n=32)$

\begin{tabular}{|c|c|c|c|c|c|c|c|c|}
\hline & Median SMI (IQR) & $p^{*}$ & Median MA (IQR) & $p^{*}$ & Median VATI (IQR) & $p^{*}$ & Median SATI (IQR) & $p^{*}$ \\
\hline \multicolumn{2}{|l|}{ Age } & 0.3 & & 0.9 & & 0.6 & & 0.008 \\
\hline$<55$ years & $42.9(39.9-47.8)$ & & $41.8(40.3-46.2)$ & & $93.5(71.7-119.0)$ & & $21.5(11.3-33.2)$ & \\
\hline$\geq 55$ years & $46.7(42.0-51.5)$ & & $32.8(27.6-41.8)$ & & $84.3(57.7-135.0)$ & & $55.5(33.3-77.7)$ & \\
\hline \multicolumn{2}{|l|}{ BMI } & - & & 0.04 & & - & & - \\
\hline$<25 \mathrm{~kg} / \mathrm{m}^{2}$ & - & & $41.8(40.5-44.5)$ & & - & & - & \\
\hline$\geq 25 \mathrm{~kg} / \mathrm{m}^{2}$ & - & & $35.4(32.1-43.8)$ & & - & & - & \\
\hline \multicolumn{2}{|c|}{ Risk for malnutriation by RMNST } & 0.9 & & 0.6 & & 0.8 & & 0.9 \\
\hline Low risk & $44.8(39.7-49.9)$ & & $40.9(30.9-46.0)$ & & $82.4(67.3-110.5)$ & & $31.2(11.9-50.8)$ & \\
\hline Moderate risk & $43.3(41.2-47.6)$ & & $34.3(29.4-43.1)$ & & $94.2(57.6-114.7)$ & & $34.9(15.0-71.6)$ & \\
\hline
\end{tabular}

SMI Skeletal Muscle Index $\left(\mathrm{cm}^{2} / \mathrm{m}^{2}\right), M A$ Muscle Attenuation (Hounsfield Units), VATI Visceral Adipose Tissue Index $\left(\mathrm{cm}^{2} / \mathrm{m}^{2}\right), S A T I$ Subcutaneous Adipose Tissue Index $\left(\mathrm{cm}^{2} / \mathrm{m}^{2}\right), B M I$ Body Mass Index, RMNST Royal Marsden Nutritional Screening Tool

*Mann-Whitney-U-Test 
Fig. 1 Examples for sarcopenia measurements in patients with high and low muscle attenuation. $\mathbf{A}$ and $\mathbf{B}$ depict examples for patients with normal muscle attenuation (MA): A 68 years, BMI $17.8 \mathrm{~kg} / \mathrm{m}^{2}$, MA 42.3 Hounsfield units (HU); B 54 years, BMI $20.4 \mathrm{~kg} / \mathrm{m}^{2}$, MA 41.8 HU; C and D depict examples for patients with low MA: C 69 years, BMI $32.9 \mathrm{~kg} / \mathrm{m}^{2}$, MA 27.6 HU; D 73 years, BMI $24.8 \mathrm{~kg} / \mathrm{m}^{2}$, MA $24.8 \mathrm{HU}$

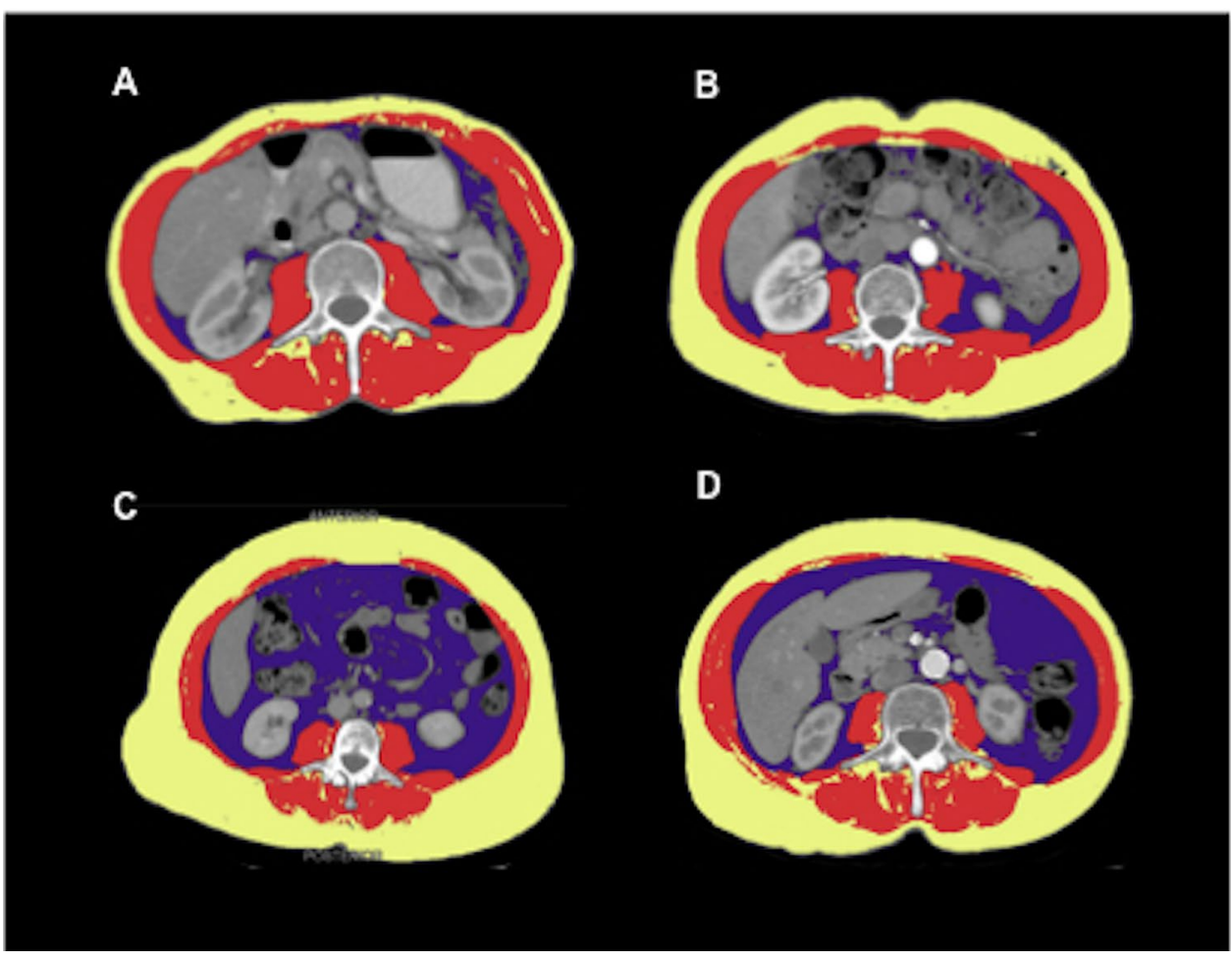

Table 3 Predictive value of clinico-laboratory parameters and body composition measurements for severe 60 days postoperative morbidity (Clavien Dindo $\geq$ grade 3 )

\begin{tabular}{lll}
\hline & $p$ & HR $(95 \% \text { CI })^{\mathrm{a}}$ \\
\hline Age $<55$ / $\geq 55$ years & 0.2 & $1.03(0.9-1.1)$ \\
ECOG $<2$ vs. $\geq 2$ & 0.4 & $0.4(0.06-2.9)$ \\
AACCI $^{\text {b }}$ & 0.2 & $2.2(0.6-9.0)$ \\
Radiotherapy in past (yes/no) & 0.5 & $1.5(0.4-5.7)$ \\
BMI $\geq 25$ vs. $<25$ & 0.2 & $2.5(0.6-9.4)$ \\
Risk for malnutrition by RMNST ${ }^{c}$ & 0.6 & $0.7(0.1-4.0)$ \\
Serum albumin & 0.5 & $1.05(0.9-1.2)$ \\
PN received post surgery & 0.1 & $0.4(0.1-1.4)$ \\
SMI $<$ vs. $\geq 41 \mathrm{~cm}^{2} / \mathrm{m}^{2}$ & 0.7 & $0.7(0.1-4.2)$ \\
MA $<$ vs. $\geq 31 \mathrm{HU}^{2}$ & 0.9 & $1.02(0.1-6.5)$ \\
VATI $\geq$ vs. $<120 \mathrm{~cm}^{2} / \mathrm{m}^{2}$ & 0.8 & $0.6(0.1-3.2)$ \\
SATI $\geq$ vs. $<57 \mathrm{~cm}^{2} / \mathrm{m}^{2}$ & $-{ }^{\mathrm{d}}$ & - \\
\hline
\end{tabular}

$H R$ Hazard Ratio, BMI Body Mass Index, $P N$ parenteral nutrition, MA Muscle Attenuation, HU Hounsfield Unit, SMI Skeletal Muscle Index, SATI Subcutaneous Adipose Tissue Index, VATI Visceral Adipose Tissue Index

${ }^{a}$ Logistic regression analyses

${ }^{\mathrm{b}}$ Age Adjusted Charlson Comorbidity Index 0-4 vs. 5-7;

${ }^{c}$ Royal Marsden Nutritional Screening Tool: moderate vs. low risk for malnutrition

${ }^{\mathrm{d}}$ Not enough events

\section{Association of nutritional factors and sarcopenia with survival outcomes}

Median (IQR) follow-up for patients alive at last followup was 40.2 (21.6-76.7) months. Within this time, 35 patients $(53.8 \%)$ died from their cancer, 5 patients $(7.7 \%)$ from other causes. Forty patients $(61.5 \%)$ had disease progression within a median (IQR) of 8.1 (5.7-14.6) months. Median (IQR) PFS and OS were 12 (6.9-28.8) and 20 (12-39.3) months, respectively. Median one-, two-, and three-year OS estimates (SE) were 80.3\% (5.1), 50.5\% (6.6), and $40.2 \%$ (6.7). Median PFS estimates (SE) at one, two, and three years were $59.1 \%$ (6.3), $41.8 \%$ (6.6), and $29.6 \%$ (6.6).

The results of uni- and multivariate survival analyses of the association of clinico-pathological, nutritional risk factors and body composition measurements with both PFS and OS are shown in Table 4.

Figure 2 shows Kaplan-Meier curves depicting the association of MA and RMNST risk groups with OS. We found moderate risk for malnutrition based on the RMNST to be the only factor associated with shorter PFS in univariate analysis. In addition to risk for malnutrition based on the RMNST, serum albumin and a low MA $(<31 \mathrm{HU})$ were associated with shorter OS in univariate analysis. 
Table 4 Association between clinico-pathological parameters, nutritional factors and body composition measurements and survival in patients undergoing pelvic exenteration for recurrent gynaecological malignancy

\begin{tabular}{|c|c|c|c|c|c|c|}
\hline & \multirow{2}{*}{\multicolumn{2}{|c|}{$\begin{array}{l}\text { Progression-free } \\
\text { survival } \\
\text { Univariate }\end{array}$}} & \multicolumn{4}{|c|}{ Overall survival } \\
\hline & & & \multicolumn{2}{|c|}{ Univariate } & \multicolumn{2}{|c|}{ Multivariate } \\
\hline & $p$ & $\mathrm{HR}(95 \% \mathrm{CI})^{*}$ & $p$ & $\operatorname{HR}(95 \% \mathrm{CI})^{\mathrm{a}}$ & $p$ & $\operatorname{HR}(95 \% \mathrm{CI})^{\mathrm{a}}$ \\
\hline Age $<55 / \geq 55$ years & 0.5 & $0.8(0.4-1.5)$ & 0.5 & $0.8(0.4-1.5)$ & - & - \\
\hline $\mathrm{ECOG} \geq 2$ vs. $<2$ & 0.7 & $1.2(0.4-4.1)$ & 0.8 & $1.1(0.3-3.7)$ & - & - \\
\hline $\mathrm{AACCI}^{\mathrm{b}}$ & 0.7 & $0.9(0.6-1.4)$ & 0.5 & $0.9(0.6-1.3)$ & - & - \\
\hline Radiotherapy in the past & 0.1 & $2.9(0.7-11.9)$ & 0.1 & $2.8(0.7-12.3)$ & - & - \\
\hline Histological Subtype $^{c}$ & 0.4 & $0.9(0.6-1.2)$ & 0.1 & $0.8(0.6-1.1)$ & - & - \\
\hline Histological tumor size & 0.4 & $1.01(0.9-1.02)$ & 0.5 & $1.0(0.9-1.02)$ & - & - \\
\hline Lymph nodes positive & 0.3 & $0.6(0.3-1.4)$ & 0.8 & $0.9(0.3-2.6)$ & - & - \\
\hline Tumour resection margin positive & 0.01 & $0.3(0.1-0.8)$ & 0.03 & $0.3(0.1-0.9)$ & 0.2 & $0.4(0.1-1.7)$ \\
\hline $\mathrm{BMI} \geq 25$ vs. $<25$ & 0.6 & $1.3(0.6-2.8)$ & 0.9 & $0.9(0.4-2.3)$ & - & - \\
\hline Risk for malnutrition by RMNST & 0.009 & $3.7(1.3-9.9)$ & 0.006 & $0.2(0.04-0.6)$ & 0.03 & $0.13(0.02-0.9)$ \\
\hline Serum albumin & 0.06 & $0.9(0.9-1.0)$ & 0.006 & $0.9(0.8-0.9)$ & 0.2 & $0.9(0.7-1.1)$ \\
\hline $\mathrm{PN}$ received post surgery & 0.9 & $1.02(0.5-1.9)$ & 0.3 & $1.3(0.7-2.5)$ & - & - \\
\hline $\mathrm{MA} \geq \mathrm{vs} .<31 \mathrm{HU}$ & 0.5 & $1.5(0.5-4.7)$ & 0.04 & $0.3(0.1-1.9)$ & 0.02 & $0.1(0.01-1.7)$ \\
\hline $\mathrm{SMI}<\mathrm{vs} . \geq 41 \mathrm{~cm}^{2} / \mathrm{m}^{2}$ & 0.09 & $0.4(0.1-1.1)$ & 0.2 & $0.4(0.1-1.4)$ & - & - \\
\hline SATI $\geq$ vs. $<57 \mathrm{~cm}^{2} / \mathrm{m}^{2}$ & 0.8 & $1.1(0.4-3.6)$ & 0.7 & $1.2(0.4-4.0)$ & - & - \\
\hline VATI $\geq$ vs. $<120 \mathrm{~cm}^{2} / \mathrm{m}^{2}$ & 0.5 & $1.5(0.5-4.8)$ & 0.5 & $1.5(0.5-5.1)$ & - & - \\
\hline
\end{tabular}

HR Hazard Ratio, CI Confidence Interval, BMI Body Mass Index, $P N$ parenteral nutrition, MA Muscle Attenuation, $H U$ Hounsfield Unit, SMI Skeletal Muscle Index, SATI Subcutaneous Adipose Tissue Index, VATI Visceral Adipose Tissue Index

${ }^{\mathrm{a}} \mathrm{Cox}$ regression analysis

${ }^{\mathrm{b}}$ Age Adjusted Charlson Comorbidity Index 0-4 vs. 5-7

${ }^{c}$ Histological subtype of primary cancer: serous/mixed serous-mucinous vs. endometrioid vs. SCC/adenosquamous vs. leiomyosarcoma/endometrial stromal sarcoma

${ }^{\mathrm{d}}$ Royal Marsden Nutritional Screening Tool (moderate vs. low risk for malnutrition)
In a multivariate analysis that adjusted for the effects of all factors associated with survival in univariate analyses, MA and moderate risk for malnutrition based on the RMNST remained independently associated with shorter OS.

\section{Discussion}

Preoperative prediction of postoperative morbidity and survival in patients undergoing major surgery, including pelvic exenteration, is essential for patient selection to assure optimal outcomes. To our knowledge, this is the first study to determine if malnutrition and sarcopenia impact on postoperative morbidity and survival in patients with recurrent gynaecological malignancies treated with pelvic exenteration. Our results demonstrate that low MA and moderate risk for malnutrition, based on the RMNST, are independent prognostic factors for survival in these patients.

The current eligibility for selection of patients with a recurrent gynaecological malignancy to undergo pelvic exenteration is fundamentally based on the pre-operative assessment that the disease is limited to the pelvis and can therefore be completely removed by surgical resection. However, only $20-50 \%$ of patients, depending on the type of primary cancer site, reportedly have a substantial benefit with long-term survival improvement [10]. Sarcopenia, a key component of cachexia, has been shown to be associated with decreased survival in various malignancies [12-16]. In ovarian cancer, few studies have investigated the effects of sarcopenia and found that low MA and SMI were associated with shorter OS $[15,16,30]$.

We found that low MA was an independent predictor for shorter OS in patients treated with pelvic exenteration for recurrent gynaecological malignancies. Current research suggests that loss of muscle quality compared to loss of muscle mass has a greater negative impact on muscle function [20]. Various molecular mechanisms leading to energywasting and loss of myofibrillar proteins in skeletal muscle cells, including the impaired function of skeletal muscle mitochondria and the secretion of inflammatory cytokines by immune or tumour cells, have been shown to result from the presence of malignancy [19]. 


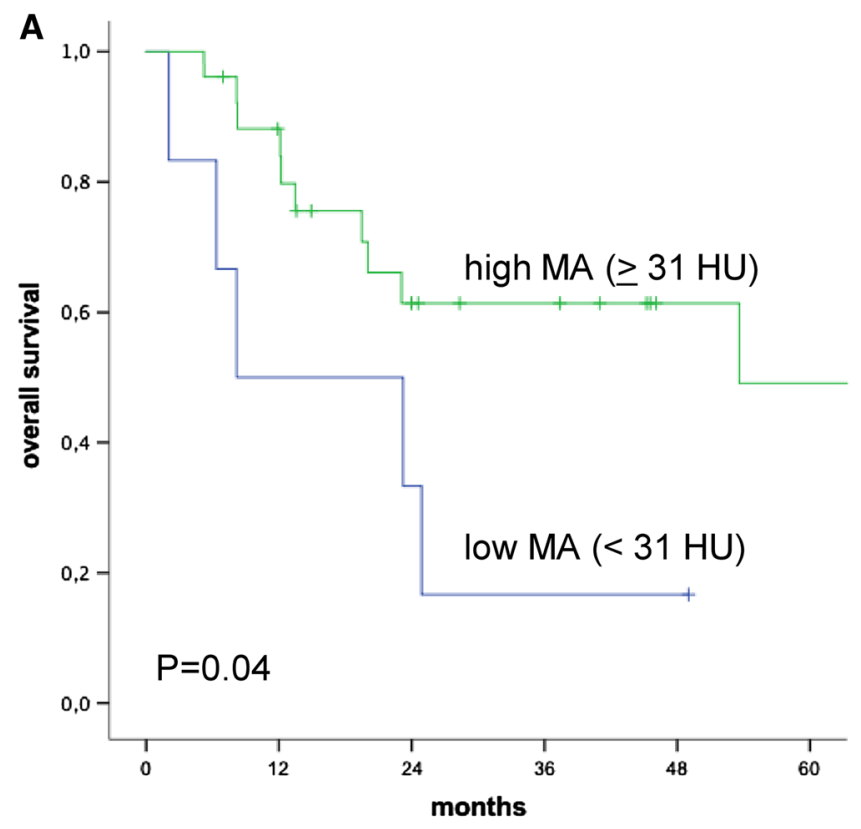

Fig. 2 Kaplan-Meier curves for overall survival. Kaplan-Meier curves depicting overall survival in patients treated with pelvic exenteration for gynaecological malignancies, stratified according to (A)

The median MA and SMI of our study population were similar to results reported for advanced ovarian cancer patients, but substantially higher than in patients with pancreatic cancer [15, 31]. Taking into account, that all patients in the present study had recurrent, often heavily pretreated disease, the comparably favourable sarcopenia measurements may attest to the patient selection process for pelvic exenteration at our institution.

Another interesting finding was the correlation between MA and the patient's BMI. In contrast to historical assumptions of cachexia (and hence sarcopenia) affecting only patients with low BMI, our results suggest a higher risk for sarcopenia in overweight and obese patients. We believe this to be an important clinical observation. These findings correlate with previously reported associations between obesity and sarcopenia and underline the risk of underdiagnosing sarcopenia when relying on BMI or overall weight loss only $[12,32]$.

In addition to radiological sarcopenia measurements, we evaluated the accuracy of the RMNST, a validated questionnaire, used as a screening tool for nutritional status [28]. In obese patients the questionnaire may be detecting weight loss-and it has been reported that even on those patients with a high BMI, weight loss can be a risk factor for poorer outcome [11]. We demonstrated that moderate malnutrition was an independent marker for both PFS and OS in our study group of patients. Interestingly, none of the patients had risk for severe malnutrition according to the RMNST. This is

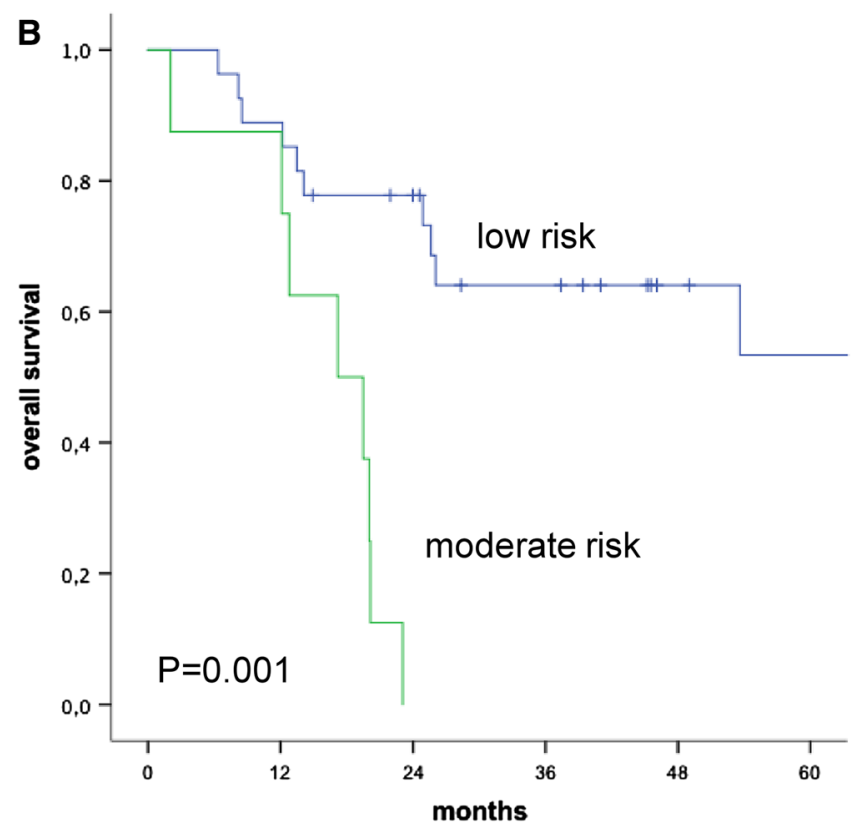

muscle attenuation (MA) and $\mathbf{B}$ risk for malnutrition according to the Royal Marsden Nutrition Screening Tool criteria

not unexpected as only patients estimated to be fit enough to undergo pelvic exenteration were selected for surgery.

Interestingly, neither radiological body composite measurements nor the risk of malnutrition according to the RMNST were associated with postoperative morbidity. In contrast to our results, sarcopenia was reported to be a risk factor for postoperative morbidity after pancreaticoduodenectomy and after gastrectomy [22, 23, 31, 33, 34]. The different outcomes of our study might be either due to the relatively small sample size or to a patient selection bias. Surgical outcome in our study in terms of median intraoperative blood loss, length of hospital stay, and postoperative morbidity were similar to those previously reported [3-6, $9,10]$. In coherence with other studies, $\mathrm{R} 0$ resection had a strong impact on progression free and overall survival [35]. However, when performing multivariable analysis only MA and risk of malnutrition according to the RMNST were independently associated with overall survival. Considering the small and heterogenous sample size this finding has to be interpreted with caution.

The main limitations of our study are its retrospective design and the relatively small sample size. Patients were only included if CT scans prior to pelvic exenteration were available for analysis, potentially causing a selection bias. A cohort of 32 patients is too low to draw a precise conclusion. However, taking into consideration of the scarcity of patients, who underwent pelvic exenteration for recurrent disease, it will be difficult to perform similar analyses within 
a bigger and more homogenous patients' cohort. Furthermore, there is not yet a standardized threshold value for SMI nor precisely defined threshold values for SMI and MA. As our study was retrospective, we could not combine SMI or MA with a functional muscle assessment. Furthermore, multiple statistical tests were performed, increasing the risk of committing type 1 errors.

We hope our results will be regarded as hypothesis generating. Further prospective studies, which should include functional muscle assessment are needed to validate these results in a larger cohort of patients undergoing pelvic exenteration.

\section{Conclusions}

In summary, our results demonstrate, for the first time, that reduced MA and malnutrition as assessed by the RMNST questionnaire are associated with reduced survival in patients treated with pelvic exenteration for recurrent gynaecological malignancies. As CT scans are performed routinely prior to pelvic exenteration, the measurement of MA could easily be incorporated in the pre-operative assessment of these patients. Especially for the planning of palliative exenteration, this could add value to appropriate patient selection. If our results are validated in larger controlled studies, these parameters could be used in clinical decision making, case selection and counselling of patients.

Supplementary Information The online version contains supplementary material available at https://doi.org/10.1007/s00404-021-06273-7.

Acknowledgements Dr. Sohaib and Prof. Rockall are in part supported by RMH/ICR NIHR Biomedical Research Centre Funding.

Author contribution VS: project development, protocol development, data collection, data analysis, manuscript writing and editing. AR: project development, protocol development, data analysis, manuscript writing. MN: project development, data management. SAS: project development, data management. TK: data analysis, manuscript writing. RA: data collection, manuscript writing. DK: data management, manuscript writing. JHS: data management, manuscript writing. CS: manuscript writing. DPB: project development, protocol development, data analysis, data management, manuscript writing.

Funding Open access funding provided by Medical University of Vienna.

\section{Declarations}

Conflict of interest We declare that we have no conflict of interest.

Ethical approval The study has been carried out with the approval of the appropriate ethical committee, the Committee for Clinical Research of the Royal Marsden Hospital NHS Foundation Trust. The committee waived the requirement to obtain informed consent.
Open Access This article is licensed under a Creative Commons Attribution 4.0 International License, which permits use, sharing, adaptation, distribution and reproduction in any medium or format, as long as you give appropriate credit to the original author(s) and the source, provide a link to the Creative Commons licence, and indicate if changes were made. The images or other third party material in this article are included in the article's Creative Commons licence, unless indicated otherwise in a credit line to the material. If material is not included in the article's Creative Commons licence and your intended use is not permitted by statutory regulation or exceeds the permitted use, you will need to obtain permission directly from the copyright holder. To view a copy of this licence, visit http://creativecommons.org/licenses/by/4.0/.

\section{References}

1. Brunschwig A (1948) Complete excision of pelvic viscera for advanced carcinoma: a one-stage abdominoperineal operation with end colostomy and bilateral ureteral implantation into the colon above the colostomy. Cancer 1:177-183

2. Rutledge FN, Smith JP, Wharton JT, O'Quinn AG (1977) Pelvic exenteration: analysis of 296 patients. Am J Obstet Gynecol 129:881-892

3. Berek JS, Howe C, Lagasse LD, Hacker NF (2005) Pelvic exenteration for recurrent gynecologic malignancy: survival and morbidity analysis of the 45 year experience at UCLA. Gynecol Oncol 99:153-159

4. Höckel M, Dornhöfer N (2006) Pelvic exenteration for gynaecological tumours: achievements and unanswered questions. Lancet Oncol 7:837-847

5. Goldberg GL, Sukumvanich P, Einstein MH et al (2006) Total pelvic exenteration: the Albert Eintstein college of medicine/montefiore medical center experience (1987 to 2003). Gynecol Oncol 101:261-268

6. Ungar L, Palfalvi L, Novak Z (2008) Primary pelvic exenteration in cervical cancer patients. Gynecol Oncol 111:S9-S12

7. Graves S, Seagle BL, Strohl AE et al (2017) Survival after pelvic exenteration for cervical cancer. A national cancer database study. Int J Gynecol Cancer 27:390-395. https://doi.org/10.1097/IGC. 0000000000000884

8. Seagle BL, Dayno M, Strohl AE et al (2016) Survival after pelvic exenteration for uterine malignancy: a national cancer data base study. Gynecol Oncol 143:472-478

9. Dessole M, Petrillo M, Lucidi A et al (2016) Quality of life in women after pelvic exenteration for gynecological malignanciesa multicentric study. Int J Gyn Cancer 28:267-273. https://doi.org/ 10.1097/IGC.0000000000000612

10. Westin S, Rallapalli V, Fellman B et al (2014) Overall survival after pelvic exenteration for gynecologic malignancy. Gynecol Oncol 134:546-551

11. Martin L, Birdsell L, MacDonald N et al (2013) Cancer cachexia in the age of obesity: skeletal muscle depletion is a powerful prognostic factor, independent of body mass index. J Clin Oncol 31:1539-1547

12. Prado CM, Lieffers JR, McCargar LJ et al (2008) Prevalence and clinical implications of sarcopenic obesity in patients with solid tumours of the respiratory and gastrointestinal tracts: a population-based study. Lancet Oncol 9:629-635. https://doi.org/10. 1016/S1370-2045(08)70153-0

13. Shachar SS, Williams GR, Muss HB, Nishijima TF (2016) Prognostic value of sarcopenia in adults with solid tumours: a metaanalysis and systematic review. Eur J Cancer 57:58-67

14. Hirasawa Y, Nakashima J, Yunaiyama D et al (2016) Sarcopenia as a novel preoperative prognostic predictor for survival in 
patients with bladder cancer undergoing radical cystectomy. Ann Surg Oncol 23:1048-1054

15. Aust S, Knogler T, Pils D et al (2015) Skeletal muscle depletion and markers for cancer cachexia are strong prognostic factors in epithelial ovarian cancer. PLoS One 10:e0140403

16. Bronger H, Hederich P, Hapfelmeier A et al (2016) Sarcopenia in advanced serous ovarian cancer. Int J Gynecol Cancer 27:223232. https://doi.org/10.1097/IGC.0000000000000867

17. Daly LE, Power DG, O'Reilly A et al (2017) The impact of body composition parameters on ipilimumab toxicity and survival in patients with metastatic melanoma. Br J Cancer 116:310-317. https://doi.org/10.1038/bjc.2016.431

18. Fearon K, Strasser F, Anker SD et al (2011) Definition and classification of cancer cachexia: an international consensus. Lancet Oncol 12:489-495

19. Argilés JM, Busquets S, Stemmler B, López-Soriano FJ (2014) Cancer cachexia: understanding the molecular basis. Nat Rev Cancer 14:754-762

20. Cruz-Jentoft AJ, Baeyens JP, Bauer JM et al (2010) European working group on sarcopenia in older patients. Sarcopenia: European consensus on definition and diagnosis: report of the European working group on sarcopenia in older people. Age Ageing 39:412-423

21. Prado CM, Baracos VE, McCargar LJ et al (2009) Sarcopenia as a determinant of chemotherapy toxicity and time to tumor progression in metastatic breast cancer patients receiving capecitabine treatment. Clin Cancer Res 15:2920-2926

22. Lou N, Chi CH, Chen XD et al (2017) Sarcopenia in overweight and obese patients is a predictive factor for postoperative complication in gastric cancer: a prospective study. Eur J Surg Oncol 43:188-195

23. Nishida Y, Kato Y, Kudo M et al (2016) Preoperative sarcopenia strongly influences the risk of postoperative pancreatic fistula formation after pancreaticoduodenectomy. J Gastrointest Surg 20:1586-1594

24. Heath OM, Bryan SJ, Sohaib A, Barton DPJ (2021) Laparoscopic assessment improves case selection for exenterative surgery in recurrent cervical and endometrial cancer. J Obstet Gynaecol 1:1-9. https://doi.org/10.1080/10443615.2020.1867963

25. American Society of Anesthesiologists. ASA physical status classification system. https://www.asahq.org/resources/clinical-infor mation/asa-physical-status-classification-system. Accessed on $12 / 12 / 2016$.

26. Chang CM, Yin WY, Wie CK et al (2016) Age-adjusted chalson comorbidity index score as a risk measure of perioperative mortality before cancer surgery. PLoS One 11:e0148076. https://doi.org/ 10.1371/journal.pone.0148076

27. Clavien PA, Barkun J, De Oliveira ML et al (2009) The ClavienDindo classification of surgical complications. Five-year experience. Ann Surg 250:187-196

28. Shaw C, Fleuret C, Pickard JM et al (2015) Comparison of a novel, simple nutrition screening tool for adult oncology inpatients and the malnutrition screening tool (MST) against the patient-generated subjective global assessment (PG-SGA). Support Care Cancer 23:47-54. https://doi.org/10.1007/s00520-014-2319-8 (Epub 2014 Jun 20)

29. Mourtzakis M, Prado CMM, Lieffers JR et al (2008) A practical and precise approach to quantification of body composition in cancer patients using computed tomography images acquired during routine care. Appl Physiol Nutr Metab 33:997-1006

30. Kumar A, Moynagh MR, Multinu F et al (2016) Muscle composition measured by CT scan is a measurable predictor of overall survival in advanced ovarian cancer. Gynecol Oncol 142:311-316

31. Van Dijk DPJ, Bakens MJAM, Coolsen MME et al (2016) Low skeletal muscle radiation attenuation and visceral adiposity are associated with overall survival and surgical site infections in patients with pancreatic cancer. J Cachexia Sarcopenia Muscle. https://doi.org/10.1002/jcsm.12155

32. Batsis JA, Mackenzie TA, Barre LK et al (2014) Sarcopenia, sarcopenic obesity and mortality in older adults: results from the national health and nutrition examination survey III. Eur J Clin Nutr 68:1001-1007

33. Pecorelli N, Carrara G, De Cobelli F et al (2016) Effect of sarcopenia and visceral obesity on mortality and pancreatic fistula following pancreatic cancer surgery. BJS 103:434-442

34. Chen FF, Zhang FY, Zhou XY et al (2016) Role of frailty and nutritional status in predicting complications following total gastrectomy with D2 lymphadenectomy in patients with gastric cancer: a prospective study. Langenbecks Arch Surg 401:813-822

35. Pelv Ex Collaborative (2019) Pelvic exenteration for advanced nonrectal pelvic malignancy. Ann Surg 270:899-905. https://doi. org/10.1097/SLA.0000000000003533

36. Seebacher V, Nobbenhuis M, Rockall A, Sohaib A, Shepherd J, Barton D (2017) Präoperative mangelernährung und sarkopenierisikofaktoren für ein kürzeres überleben von patientinnen nach pelviner exenteration? Geburtshilfe Frauenheilk 77(04):396-405

37. Seebacher V, Rockall A, Nobbenhuis M, Sohaib A, Knogler T, Alvarez-Lopez R, Kolomainen D, Shaw C, Shepherd J, Barton DP (2017) Malnutrition and sarcopenia: risk factors for shorter survival after pelvic exenteration for recurrent gynecological malignancy. Proceedings of the international cancer imaging society (ICIS) 17th annual teaching course, Berlin, Germany, 02-04 October 2017. Cancer Imaging 17(Suppl 1):24 (Published online 2017 Sep 7)

38. Seebacher V, Rockall A, Nobbenhuis M, Lopez RMA, Sohaib A, Knogler T, Barton D (2017) Malnutrition and sarcopenia: risk factors for shorter survival in patients treated with pelvic exenteration for recurrent gynecological malignancy. 20th International Meeting of the European Society of Gynaecological Oncology, Vienna, Austria, November 4-7, 2017 Int J Gyn Cancer 27(Suppl 4)

Publisher's Note Springer Nature remains neutral with regard to jurisdictional claims in published maps and institutional affiliations. 


\section{Authors and Affiliations}

\section{Veronika Seebacher ${ }^{1,2}$ (1) - Andrea Rockall ${ }^{3}$ Marielle Nobbenhuis ${ }^{1} \cdot$ S. Aslam Sohaib ${ }^{3}$. Thomas Knogler ${ }^{4}$.} Rosa M. Alvarez ${ }^{1}$. Desiree Kolomainen ${ }^{5}$. John H. Shepherd ${ }^{1}$. Clare Shaw ${ }^{6}$. Desmond P. Barton ${ }^{1}$

1 Department of Gynaecological Oncology, The Royal Marsden NHS Foundation Trust, 203 Fulham Road, Chelsea, London SW3 6JJ, UK

2 Department of Gynaecology and Gynaecologic Oncology, Medical University of Vienna, Waehringer Guertel 18-20, 1090 Vienna, Austria

3 Department of Radiology, The Royal Marsden NHS Foundation Trust, 203 Fulham Road, Chelsea, London SW3 6JJ, UK
4 Department of Biomedical Imaging and Image-Guided Therapy, Medical University of Vienna, Waehringer Guertel 18-20, 1090 Vienna, Austria

5 Department of Gynaecologial Oncology, King's College Hospital NHS Foundation Trust, Denmark Hill, London SE5 9RS, UK

6 Department of Nutrition and Dietetics, The Royal Marsden NHS Foundation Trust, Downs Road, Sutton SM2 5PT, UK 